Claves. Revista de Historia, Vol. 2, $\mathrm{N}^{\mathrm{O}} 3$

Montevideo, Julio-Diciembre 2016

(pp. 133-161) ISSN 2393-6584

\title{
La geopolítica de la radiodifusión: Estados Unidos y la radio latinoamericana durante la Segunda Guerra Mundial*
}

\author{
Gisela Cramer \\ Universidad Nacional de Colombia, Sede Bogotá
}

Recibido: 10/11/2016

Aceptado: 12/12/2016

\section{Resumen}

Hacia comienzos de la Segunda Guerra Mundial, y en medio de una crisis de seguridad nacional profunda, el gobierno estadounidense desplegó una series de políticas destinadas a influir sobre los medios de comunicación latinoamericanos con el fin de asegurar la lealtad y cooperación latinoamericanas. Por razones de espacio, el presente ensayo se concentra en la radio. Además de brindar una revisión de tales políticas, sus objetivos y modos de implementación, se analiza el impacto en distintos escenarios nacionales para visibilizar interacciones complejas entre actores estatales y privados que reforzaron o delimitaron el alcance del poder estadounidense sobre los medios radiofónicos y los empresarios de la radio en América Latina.

\section{Palabras clave}

Historia de la radio; relaciones interamericanas; Cadenas Panamericanas; retransmisiones transcontinentales

\section{The Geopolitics of Broadcasting: The United States and Latin American Radio during World War II*}

\begin{abstract}
Toward the beginning of World War II, and in the midst of a profound national security crisis, the US government deployed a series of policies that were meant to influence Latin America's media with a view to securing Latin American allegiance and cooperation. For reasons of space, this essay concentrates on radio. Apart from providing a panoramic overview over these policies, their objectives, and means of
\end{abstract}


implementation, it analyzes their impact in different national scenarios, in order to visualize complex interactions between state and private actors, which enhanced or constrained US power over Latin America's broadcasting and radio entrepreneurs.

\section{Keywords}

Radio history; inter-American relations; Pan-American networks; transcontinental retransmissions

Este ensayo trata sobre una coyuntura particular de la historia de la radiodifusión latinoamericana ubicada en los años finales de la década de 1930 y durante la Segunda Guerra Mundial. Así como sucedió en otras partes del mundo en este contexto, el paisaje sonoro latinoamericano se tornó en un campo de acalorada disputa, en tanto todas las potencias mayores (y varias de las menores) del mundo competían por alcanzar audiencias masivas más allá de sus fronteras. En este contexto, la radio parecía establecerse como una prometedora herramienta dentro del arsenal propagandístico del momento. Si bien, no todos los hogares latinoamericanos estaban equipados con una radio, ésta ya alcanzaba a millones de oyentes, alfabetizados o no.

Por razones de espacio este ensayo se concentra en los esfuerzos del gobierno y la industria radiofónica de Estados Unidos para movilizar la radiodifusión como una herramienta de política exterior para obtener la cooperación latinoamericana frente a la inminente guerra. Se analizan las principales estrategias empleadas y la manera en que estas evolucionaron durante los años de guerra. Se estudia también el impacto de tales estrategias focalizando el estudio en Argentina, Uruguay, México, Cuba y Brasil, para visibilizar interacciones complejas entre actores estatales y privados que reforzaron o delimitaron el alcance del poder estadounidense sobre los medios radiofónicos y los empresarios de la radio latinoamericanos. Aquí se evidencia el poder de Estados Unidos y su habilidad de moldear los paisajes sonoros latinoamericanos en un momento crítico de la historia, al tiempo que se señalan los límites de su hegemonía. Cualquier intento de alcanzar audiencias masivas dependía de la colaboración de empresarios y gobiernos latinoamericanos, y ésta no se dio de la misma manera en todas partes, como se verá en las páginas que siguen. 


\section{La carrera por la supremacía en la onda corta}

Hacia finales de la década de los años treinta, políticos estadounidenses observaban con creciente preocupación la incapacidad de su país por establecer un servicio radiofónico internacional a la par de su estatus de potencia mundial. La industria de radiodifusión estadounidense había sido lenta a la hora de desarrollar ofertas para audiencias extranjeras, es decir, servicios de onda corta porque atraían a pocos patrocinadores comerciales y producían pérdidas en vez de ganancias ${ }^{1}$. Por esta razón las emisoras comerciales se sentían poco inclinadas a invertir en sus estaciones de onda corta y mucho menos en desarrollar programas diseñados para audiencias extranjeras. En Europa en cambio se había invertido fuertemente en la onda corta, y las emisoras de la Alemania nazi alcanzaban casi todos los rincones del globo, incluyendo América Latina. De ahí que, mientras la voz del Führer podía ser escuchada fuerte y clara, en muchas partes de América Latina siguió siendo difícil sintonizar las estaciones estadounidenses. Más aún, gran parte de la programación de estas últimas solía consistir de contenidos producidos para el público local con poca consideración a radioyentes en el extranjero.

El auge de la onda corta Alemana levantó particulares preocupaciones, y no solamente en Estados Unidos. La radio, como se solía creer, había sido una de las herramientas más eficaces en manos de los nazis en su ascenso al poder. ¿Y quién podía garantizar que los talentos sugestivos de Joseph Goebbels se limitaran a los alemanes? Cierto es, estudios sistemáticos sobre el impacto de emisoras extranjeras en la opinión pública estadunidense pronto sepultaron algunos de los temores más acentuados. Estos estudios concluyeron que la onda corta en general alcanzaba a pocos de los ciudadanos estadounidenses, y que los programas alemanes en particular atraían a grupos aún más pequeños ${ }^{2}$, y aparentemente con poco efecto: "Radio Propaganda Nazi Declarada Inútil”, resumió en mayo de 1941 el diario The Washington Post unos estudios liderados por el Princeton Listening Center3. Si bien

\footnotetext{
* Agradezco el apoyo brindado por el German Historical Institute (Washington DC), el Rockefeller Archive Center, la Franklin D. Roosevelt Presidential Library y la Universidad Nacional de Colombia.

${ }^{1}$ Véase, por ejemplo, RABE, Robert, "Selling the Shortwaves: Commercial Broadcasting to Latin America and the Limits of the 'American System'," in American Journalism 24:4, pp. 127-48, 2007.

2 Véase, entre otros,. CHILDS, Harwood L, "Shortwave Listening in the United States", Public Opinion Quarterly 5, June 1941, pp. 210-226.

3 The Washington Post, 18 de mayo de 1941, p. 14.
} 
los radioyentes en Estados Unidos en su gran mayoría parecían ser impenetrables a propaganda fascista, los líderes estadounidenses se mostraban menos convencidos de la imperturbabilidad de las audiencias en América Latina, las que consideraban como emocional y políticamente inestables y, por lo tanto, más propensas a caer en la propaganda nazi. Sumado a lo anterior, América Latina era hogar de vastos núcleos de descendientes de alemanes e italianos, de quienes se temía que, alentados por Berlín y Roma se convirtieran en quinta columnas y en activos agentes del fascismo4.

El gobierno en Washington no era el único en preocuparse por la arremetida comunicacional nazi. El de Gran Bretaña, en particular, respondió con vigor. Así, los servicios en español y portugués de la British Broadcasting Corporation (en adelante BBC) y particularmente sus noticieros internacionales, pronto gozaron de un creciente prestigio en América Latina. Sin embargo, tales éxitos poco hicieron por mitigar las preocupaciones en Washington ya que se dieron en una región considerada como una zona de influencia cuasi natural de Estados Unidos. "Si bien no existe una Doctrina Monroe del aire", comentó un analista con el New York Times hacia fines de 1938, "el Tío Sam, no obstante, está aplicando algunos de sus principios a la radiodifusión”.

Para esta fecha, si bien se hablaba mucho en el Capitolio sobre la necesidad de asegurar la supremacía de EEUU en materia de onda corta, no existía consenso acerca de cómo alcanzar semejante objetivo. Un grupo de congresistas presentó un proyecto de ley exigiendo al gobierno que construyera una emisora lo suficientemente poderosa como para garantizar el liderazgo radiofónico en el hemisferio. Esta iniciativa fue rechazada enérgicamente por la industria de radiodifusión. Como se mencionó antes, los servicios internacionales arrojaban pérdidas y por lo tanto no parecían tener un valor comercial para proteger. Sin embargo, para los empresarios radiofónicos esta iniciativa constituyó una "cuña de entrada" que abriría las puertas para una intervención progresiva que tarde o temprano se extendiera hacia el corazón rentable de sus negocios. Ya que la Casa Blanca decidió no respaldar la iniciativa legislativa, los empresarios ganaron con creces esa disputa pero su victoria llegó con un precio: por medio de nuevas reglamentaciones, el gobierno ahora obligaba a los empresarios a

4 Sobre la percepción de la amenaza nazi en Estados Unidos véase FRIEDMAN, Max Paul, Nazis and Good Neighbors: The United States Campaign Against the Germans of Latin America in World War II, Cambridge, Cambridge University Press, 2003; LÜBKEN, Uwe, Bedrohliche Nähe. Die USA und die nationalsozialistische Herausforderung in Lateinamerika, 1937-1945, Stuttgart, Steiner, 2004.

5The New York Times, 11 de diciembre de 1938, p. 200. “Singing Down to Rio”, por Orrin E.Dunlap. 
mejorar sus servicios internacionales o arriesgarse a perder sus licencias de onda corta $^{6}$.

Tales mejoras, sin embargo, tardaron en materializarse. Los empresarios solían presentarse con firmeza frente a los políticos y al público en general cuando se trataba de defender el "sistema Americano" en contra del intervencionismo estatal; un sistema, insistían, que además de asegurar la libertad de opinión dentro de las fronteras del país, constituía también una ventaja en el exterior. Dejar los servicios internacionales en manos privadas, argumentaban, arrojaría una ventaja competitiva frente a la onda corta europea, ya que en todas partes los radioyentes identificarían a las emisoras estatales como órganos de propaganda. Por sí mismos, empero, los defensores del "sistema Americano" eran incapaces de superar las potentes estaciones de onda corta europeas. Estos usaban cadenas de poderosos transmisores para la transmisión simultánea de sus programas, lo que proporcionaba no sólo una señal fuerte sino también permitía a los radioescuchas cambiar a otras frecuencias en caso de interferencias atmosféricas7. En contraste, los servicios de onda corta de EE.UU. eran operados por seis corporaciones: Columbia Broadcasting System (en adelante CBS); National Broadcasting Company (en adelante NBC); General Electric; Crosley Corporation; Westinghouse; y la World Wide Broadcasting Foundation, una emisora no comercial apoyada por la Fundación Rockefeller. En su conjunto, estas emisoras ofrecían una gama más amplia de contenidos, pero esto a costa de la dispersión en términos tanto de potencia de la señal como de calidad de programas, como lo evidenciaban las encuestas encargadas por el gobierno. Muchos de los programas transmitidos por onda corta presentaban contenidos producidos para el mercado doméstico "con algunas palabras en español interpuestas a toda prisa para anunciar el título de una canción", informaba en setiembre de 1940 un funcionario consular desde

\footnotetext{
${ }^{6}$ Más información sobre las iniciativas legislativas y sus resultados se encuentra en: GREGORY, Bruce N., The Broadcasting Service: An Administrative History. The United States Information Agency Special Monograph Service, Washington, DC, USIA, 1970; FEJES, Fred, Imperialism, Media, and the Good Neighbor: New Deal Foreign Policy and United States Short Wave Broadcasting to Latin America, Norwood, NJ, Ablex, 1986, cap. 4.

7 Según estudios estadunidenses, los alemanes usaron regularmente 16 frecuencias para transmitir a América; siete de estas transmitían en inglés (para Norteamérica); seis transmitían en español y tres en portugués (para América Latina), véase: National Archives and Record Administration (NARA), Estados Unidos, College Park, MD, Record Group (RG) 229, caja 239, carpeta: Interdepartmental Committee International Radio Broadcasting Facilities), "The Program Plan for International Broadcasting," p. 1.
} 
Buenos Aires: esos programas "no significan nada para el escucha latinoamericano"s. A finales de 1942 un sondeo de la recepción de los servicios de onda corta en el Cono Sur reportaba: "No se puede sintonizar una estación de radio Norteamericana con la expectativa de por lo menos una hora de escucha interesante. O bien los programas son mediocres, la frecuencia se desvanece, [...] o la estación cambia de idioma"

Fue la intervención estatal la que cambió el panorama. Después del "relámpago" que dejó a gran parte de Europa bajo el régimen del terror de Alemania nazi, en Estados Unidos se estableció, en agosto de 1940 (lo que llegó a conocerse como) la Oficina del Coordinador de Asuntos Interamericanos (en adelante OCAIA). Dirigido por el joven y multimillonario empresario Nelson A. Rockefeller, este organismo fue encargado, entre otras cosas, de asistir a las cadenas privadas en sus esfuerzos de mejorar la calidad de sus señales y programas transmitidos. Pero hasta la OCAIA, muy cercana a la filosofía del "sistema Americano" se dio cuenta que para establecer servicios internacionales de rango mundial se requería de una reorganización profunda del sector. Por lo tanto, asintió cuando el gobierno federal, en noviembre de 1942, tomó control sobre la onda corta, arrendando a todas las estaciones existentes y reservando un tercio del total de las horas de programación para las transmisiones a América Latina bajo la responsabilidad de la OCAIA. Para nuestros fines es importante señalar que el gobierno (hasta 1948) siguió contratando a NBC y CBS para la producción y transmisión de los programas, a la vez que se dispuso la reestructuración de todo el sistema de onda corta. En cuanto a América Latina, para mediados de 1943 la OCAIA procedió a integrar todos los servicios dirigidos hacia la región en un solo sistema de radiodifusión: las Emisoras de los Estados Unidos, con CBS y NBC preparando programas en horarios alternados. Esta reorganización, por medio de una puesta en común de los recursos racionalizó el uso de las emisoras y frecuencias existentes y las franjas disponibles para la programación en español, portugués e inglés ${ }^{10}$. Junto a un masivo incremento en la capacidad física,

\footnotetext{
8 NARA, RG 229, caja 243, carpeta: Misc. Short Wave [1], Joe D. Walstrom, "Further improvements desirable in U.S. Shortwave Programs for Latin America," Sept. 1940, p. 3.

9 NARA, RG 229, caja 243, carpeta: Short wave misc [2], "Memorandum John W. G. Ogilvie to Don Francisco", June 1, 1942, p. 1.

10 Véase NARA, RG 229, caja 243, carpeta: Short wave misc. [2], "Short Waves and the CIAA Coordinated Plan of International Broadcasting to the Other Americas", pp. 1-2; Rockefeller Archive Center (RAC), Estados Unidos, RFA, RG 4, NAR Papers, Washington Files, OIAA, caja 4, carpeta 49, "Memorandum Dwight Jennings to Nelson A. Rockefeller", Nov. 29, 1944.
} 
esta unificación de las transmisores permitía a los oyentes en cualquier parte de América Latina escuchar programas en un solo idioma durante períodos de tiempo más prolongados y en diferentes bandas de frecuencia. Al mismo tiempo, el volumen de las emisiones dirigidas a América Latina seguía aumentando con un promedio de 40 horas diarias de programación para principios de $1945^{11}$.

Para esta fecha, la onda corta estadunidense había dejado muy atrás a la onda alemana en infraestructura física instalada y volumen de programación ${ }^{12}$. De hecho, desde 1943 la OCAIA y demás organismos de la política exterior, en sus reportes e informes acerca del desempeño de la onda corta estadounidense ahora la comparaban con la BBC y no con las ondas de Alemania y sus aliados ${ }^{13}$.

\section{En búsqueda de radioescuchas para la onda corta}

La capacidad para saturar el aire, sin embargo, no garantizaba que, de hecho, la onda corta iba a encontrar a muchos oyentes. Los primeros sondeos sistemáticos de los hábitos y preferencias de radioescuchas latinoamericanos realizados a inicios de 1941 en Argentina y Brasil, sugerían que, en términos comparativos, a la onda corta norteamericana le fue mejor de lo que se había esperado. Es cierto que en Brasil el conjunto de las emisoras estadounidenses alcanzaron un puntaje muy inferior a la BBC, pero tal resultado no sorprendió a nadie dado el estado rezagado de los servicios en portugués. Aun así, en conjunto parecieron atraer a más oyentes que las emisiones provenientes de Alemania o Italia ${ }^{14}$. En Argentina, y para sorpresa de la OCAIA, los radioescuchas parecían preferir programas estadounidenses, y en varios categorías incluso sobre los de la $\mathrm{BBC}^{15}$. Sin embargo, tales hallazgos poco hicieron por mitigar

${ }^{11}$ Durante los años de la guerra, el número de las emisoras de onda corta estadunidenses aumentó de 12 a 36; por más detalles véase: OGILVIE, John W. G., "The Potentialities of Inter-American Radio", Public Opinion Quarterly 9, 1, primavera 1945, pp. 19-28.

12 Véase: BOELCKE, Willi A., Die Macht des Radios. Weltpolitik und Auslandsrundfunk, 1924-1976, Frankfurt am Main, Ullstein, 1977, pp. 48-49, 58.

13 Tal preocupación reflejaba una tendencia más general, véase: NARA, RG 84, Buenos Aires Confidential Files, caja 35, carpeta 820.02 - British Information Activities in Argentina, "Strictly Confidential Circular A.A. Berle to Chiefs of Diplomatic Mission in Other American Republics", 26 de noviembre de 1943 y "Strictly Confidential Dispatch 9404 Ambassador Norman Armour to Secretary of State", 18 de marzo de 1943.

14 NARA, RG 229, Box 135, Folder: Surveys 9, A Survey of Communications in Brazil, "Lloyd Free's Report”.

15 NARA, RG 229, Box 310, Folder: Surveys, "Survey of Communications in Argentina. Confidential Report from Hadley Cantril”. 
las preocupaciones estadounidenses. La OCAIA aspiraba a una clara hegemonía en la onda corta.

La OCAIA, por lo tanto, no se limitaba a mejorar la calidad de la señal y los programas ofertados por CBS y NBC; también financió espacios publicitarios en los periódicos latinoamericanos más importantes, en los que se publicaba la programación semanal. Asimismo, buscaba ponerse en contacto directo y continuo con miles de hogares latinoamericanos. Por ejemplo, para octubre de 1944 la dependencia de la OCAIA en Buenos Aires informaba que enviaba 21.00o guías de programación destinadas a hogares de todo el país ${ }^{16}$.

No obstante, lo más importante fue el esfuerzo de producir programas más atractivos. Para ser efectivo, ese esfuerzo debía definir el tipo de radioescuchas que se buscaba atraer y la estrategia para hacerlo. Los analistas de la OCAIA asumieron que la onda corta era de uso predominantemente masculino, y sus propios informes señalaban que, así como sucedía en Estados Unidos, los radioescuchas latinoamericanos más proclives a sintonizar la onda corta internacional pertenecían más bien a capas sociales educadas y prósperas; también indicaban que la razón principal por la cual los oyentes sintonizaban estaciones extranjeras era para escuchar noticias ${ }^{17}$.

En consecuencia, el mejoramiento de los noticieros era el paso más obvio a seguir. Las noticias internacionales, sin embargo, siguieron siendo poco alentadoras para los aliados mientras el Eje triunfaba en los campos de guerra, razón por la cual la OCAIA invirtió esfuerzos considerables en programas de comentarios que, como complemento a los noticieros del día, reflexionaran sobre la fuerza y el espíritu superior de Estados Unidos y sus aliados. Como comentaristas se contrataron periodistas latinoamericanos quienes como Roberto Unanue, Fernando Ortiz Echagüe

${ }^{16}$ NARA, RG 229, caja 310, carpeta: Radio Reports, “Report on Argentine Radio”, Oct. 1944.

17 Véase, por ejemplo, "Survey of Communications in Argentina. Confidential Report from Hadley Cantril", ob.cit. 
y Alejandro Sux, para mencionar unos pocos ejemplos (argentinos), prestaron su voz de autoridad a tales programas ${ }^{18}$.

Sin embargo, la OCAIA buscaba construir audiencias más amplias, más allá de los segmentos reducidos que habitualmente sintonizaban estaciones extranjeras. Imbuidos de una cultura empresarial que veía a la radio como una herramienta para manejar un mercado de masas en vez de impresionar a unas capas sociales selectas, los oficiales de la OCAIA, en su gran mayoría procedentes de la industria radiofónica o agencias publicitarias se esforzaron a llegar a todos los sectores de la población incluyendo mujeres y jóvenes. Así pues, mientras los noticieros y programas afines cubrían importantes franjas de la programación diaria, le fue otorgado un espacio considerable a producciones teatrales, particularmente a dramas históricos, a deportes, programas de curiosidades, música popular o chismes de Hollywood. Si esta estrategia de abrir la onda corta para un espectro más amplio de oyentes ${ }^{19}$ fue exitosa o no, es difícil de asegurar, dada la naturaleza limitada de los sondeos de audiencias que se hicieron durante la guerra. Entre los intelectuales y letrados latinoamericanos, por cierto, abundaron las críticas. Por ejemplo, Hernane Tavares de Sá, denunció públicamente que la onda corta estadounidense parecía orientada hacia "unos niños imbéciles de doce años”, a la vez que festejaba a los servicios internacionales de la $\mathrm{BBC}^{20}$.

En cualquier caso, los sondeos de audiencias llevados a cabo por la OCAIA indicaron que, por lo general, la onda corta internacional alcanzó un público limitado aunque con cierta variación regional. En Argentina, donde por lo menos tres cuartos de la población adulta ya tenía acceso a la radio, los datos recabados indicaban que los oyentes sintonizaban con bastante frecuencia las estaciones extranjeras, lo que también se relaciona con la composición étnica de la población ${ }^{21}$. En otras regiones,

\footnotetext{
18 Para más detalles véase CRAMER, Gisela , "The Word War at the River Plate", CRAMER, Gisela y PRUTSCH, Ursula (ed.) iAméricas Unidas! Nelson A. Rockefeller's Office of Inter-American Affairs, 1940-46, Frankfurt y Madrid, Vervuert, 2012.

19 Un análisis comparativo de la programación de onda corta desde Alemania, Gran Bretaña, Italia y Estados Unidos se encuentra en: NARA, RG 229, caja 243, carpeta: Short-wave misc [2], "Memorandum Wells Church to Ogilvie", 4 de enero de 1943.

${ }^{20}$ The Washington Post, 10 de junio de1944, pp. 1. Cabe señalar que Tavares de Sá, además de ser un hombre público en Brasil, se desempeñó como consultor de la OCAIA.

${ }_{21}^{21}$ "Survey of Communications in Argentina. Confidential Report from Hadley Cantril", ob. cit, Tablas $8,12,21$.
} 
fuera de las zonas de inmigración europea masiva, las emisoras de ultramar parecen haber alcanzado sólo a una fracción menor de la población.

\section{Retransmisiones}

Dado que la radio de onda corta atraía a audiencias limitadas, todas las potencias mayores (y varias de las menores) procuraron contratar con emisoras locales en el exterior, para la retransmisión de programas de sus servicios de onda corta. La BBC en particular fue muy exitosa en hallar aliados. Para finales de 1942, sus boletines de noticias se retransmitieron por más de sesenta emisoras de onda media en toda América Latina22. Alemania también se había esforzado al respecto y con resultados nada despreciables. Sin embargo, el volumen de las retransmisiones alemanas pronto disminuyó notablemente por efecto de las "listas negras" ${ }_{23}$ con las que los Aliados lograron intimidar a las emisoras clasificadas como "amigables" al Eje. Entrar en las listas negras reducía oportunidades comerciales y el acceso a repuestos importados. Estados Unidos en particular tenía una capacidad muy amplia para presionar a las emisoras y a sus dueños. En muchas partes de América Latina, empresas estadounidenses como Colgate Palmolive, Sterling Products o Coca-Cola habían alcanzado un peso notable como patrocinadores de programas y anuncios comerciales. Ahora cooperaban de cerca con la política exterior se su país, retirando su patrocinio de las emisoras cuestionadas, a la vez que, alentadas por nuevas exenciones de impuestos en Estados Unidos, aumentaron el volumen global de sus inversiones en América Latina ${ }^{24}$. Además, Estados Unidos controlaba el suministro de varios de los repuestos esenciales para el funcionamiento de las emisoras, que durante la guerra eran difíciles, si no imposibles, de conseguir en otras partes.

Las embajadas alemanas respondieron ofreciendo subsidios compensatorios a aquellas estaciones que siguieran retransmitiendo programas del Reich, pero tales esfuerzos tuvieron efectos limitados. Aún en Argentina, donde podían operar con más

${ }^{22}$ BBC Year-Book (1943), p. 20.

23 Sobre el impacto del "blacklisting" véase los casos de Radio Callao y Fénix en Buenos Aires, detallados en: NARA, RG 84, Buenos Aires Embassy Confidential File, Box 25, Folder 876 1942, "Geoffrey Wallinger, British Embassy Buenos Aires, to Joseph Walstrom, U.S. Embassy", 07.04.1942; y "Joseph Walstrom to Geoffrey Wallinger", 10 de abril de 1942.

24 La OCAIA había hecho lobby para tales exenciones; véase: Julio Moreno, Yankee Don't Go Home: Mexican Nationalism, American Business Culture, and the Shaping of Modern Mexico, 1920-1950 (Chapel Hill, NC: University of North Carolina Press, 2003), pp. 46, 71-72. 
libertad que en muchas otras partes, los propagandistas alemanes tenían que limitarse a emisoras más bien pequeñas y débiles ${ }^{25}$, varias de las cuales estaban a la vez al servicio de los Aliados. Incluso Radio Prieto, la "columna vertebral" de las radio operaciones alemanas en la región del Río de la Plata que consumía gran parte de los fondos disponibles para fines de propaganda, transmitía simultáneamente noticieros de la $\mathrm{BBC}^{26}$.

\section{Las cadenas Panamericanas}

Estados Unidos, por lo tanto, no era de manera alguna el único interesado en usar emisoras locales para la retransmisión, pero la industria radiofónica estadunidense puso en marcha una estrategia mucho más ambiciosa: la incorporación de las mejores estaciones latinoamericanas en las cadenas comerciales de Estados Unidos. La CBS lideró la corrida expansionista cuando en 1940 comenzó a buscar socios para su nueva Cadena de las Américas. Sus competidores pronto siguieron su ejemplo, dentro de ellos la NBC que estableció lo que llegó a ser la más extensa de las cadenas transcontinentales, la Cadena Panamericana. Se esperaba que las estaciones miembros retransmitieran un creciente volumen de los programas de onda corta que CBS, NBC y otros producían para las audiencias latinoamericanas y compartir los ingresos producto de publicidad.

La retransmisión por medio de emisoras potentes prometía acceso a audiencias masivas y, por lo tanto, más posibilidades para atraer a las grandes corporaciones con intereses comerciales en la región como patrocinadores. Sin embargo, el más interesado resultó ser el gobierno en Washington. La OCAIA, en particular, trazó grandes esperanzas en las nuevas cadenas transcontinentales. Sus sondeos del panorama radiofónico latinoamericano le habían dejado con la impresión que la calidad de dicha radio era muy inferior a los estándares norteamericanos, razón por la cual sus oficiales, al comienzo, se dejaron llevar por una visión muy optimista acerca del impacto que pudieran tener programas preparados por la mano experta de empresas como CBS o NBC. La OCAIA, por lo tanto, patrocinó no solamente la

\footnotetext{
25 La única excepción fue la red de Radio Splendid, que en 1936 retransmitió para su resumen diario de noticias internacionales, unos tres minutos de noticias desde Berlín, junto con los resumes llegados de Italia, Francia (reemplazada por Gran Bretaña después de 1940) y de Estados Unidos. Por tratarse de una emisora clasificada como "pro-aliada", Radio Splendid no sufrió inconvenientes.

${ }^{26}$ Más información se encuentra en: CRAMER, Gisela "Der Krieg im Äther”, ob. cit.
} 
producción sino también la colocación de programas con las emisoras asociadas de la CBS y NBC. Al apoyar la expansión de las grandes cadenas hacia el sur del continente, explicaba un memorando interno de 1941, la OCAIA iba a revolucionar el panorama radiofónico latinoamericano y, a la vez, profundizar la influencia estadounidense en la región: "Haremos de la radio no sólo más deseable a partir de la calidad de los programas ofertados, sino también más interesante, más variada, más colorida [...] y mientras crecen nuestras audiencias, tendremos los medios para educar, influir e informar las gentes de las otras Repúblicas [Americanas]”27.

La respuesta latinoamericana, en un principio, pareció confirmar este optimismo. Para finales de 1941, de acuerdo con las estimaciones de Fred Fejes, aproximadamente un tercio de las estaciones latinoamericanas se habían afiliados con las cadenas estadounidenses28. Más importante aún, la mayoría de los empresarios más exitosos de las radiocomunicaciones se afiliaron, incluyendo a Emilio Azcarrága (México), Jaime Yankelevich y Benjamín Gache (Argentina), empresarios que habían construido redes extensas en sus respectivos países e incluso más allá. Igualmente se unieron las dos cadenas más fuertes de Cuba, CMQ y RHC. Con la afiliación llegaron a ser retransmitidos una vasta variedad de contenidos estadounidenses, por lo general adaptaciones de programas exitosos como "The Hit Parade" (con las canciones populares más exitosas del momento); "The March of Time" (noticias internacionales en formato dramatizado); "The World is Yours" (sobre los avances de las ciencias) o "Counterspy" (un thriller de espionaje).

\section{Retransmisiones en búsqueda de audiencias masivas}

Hacia comienzos de 1943, sin embargo, se hizo cada vez más claro que la realidad de las nuevas cadenas transcontinentales distaba mucho de las expectativas optimistas. Las emisoras latinoamericanas se mostraban más bien reacias cuando se trataba de incorporar programas producidos en Estados Unidos y más aún cuando se les solicitó reservar horarios de alta sintonía para las retransmisiones. Debido al hecho que la onda corta era (y es) propensa a interferencias atmosféricas ("fadings"), los programas llegaban en baja calidad, sobre todo aquellos contenidos que (como

${ }_{27}$ NARA, RG 229, caja 241, carpeta: Procedure (misc), "Memorandum Communications Division to Nelson A. Rockefeller“, 4 de octubre de 1941, p. 5.

${ }^{28}$ FEJES, Fred, ob. cit., p. 129. 
programas de música o el radioteatro) desplegaban un espectro sonoro más complejo. Por esta razón muchos empresarios que operaban en entornos competitivos y que dependían del ratings insistieron cada vez más en que tales contenidos se les fueran entregados por medio de grabaciones (transcripciones eléctricas) y no recibidos a través de onda corta. Las grabaciones brindaban un sonido prístino y ofrecían flexibilidad a la hora de la programación ya que se podían integrar más fácilmente dentro de los horarios establecidos. El uso creciente de grabaciones, sin embargo, no resolvió todos los obstáculos en la formación de las cadenas transcontinentales

En lugar de concentrarse en los noticieros y programas afines o música, las emisoras cabeceras en Estados Unidos, bajo el creciente control de la OCAIA, insistieron en suministrar una amplia variedad de contenidos más abiertamente propagandísticos a sus afiliados latinoamericanos. Insistieron, sobre todo, en la retransmisión de géneros dramáticos. Para la OCAIA, los géneros dramáticos, con sus despliegues de tecnología de punta en cuanto a los efectos sonoros prometían ser vehículos idóneos para inspirar la solidaridad panamericana bajo la sombrilla protectora de Estados Unidos. Con este fin se iniciaron las adaptaciones al español y portugués del programa "The March of Time” (en su versión español, "La marcha del tiempo") como una suerte de guía exegética puesto para poner en orden el convulsionado mundo de las noticias internacionales. Adaptaciones de seriales como "This is War" ("Estamos en guerra") retrataban los objetivos estadounidenses en la contienda mundial y la moral inquebrantable de su pueblo a pesar de privaciones crecientes. El destino de los intelectuales europeos bajo el nazismo se ilustraba en antologías como "Las ideas no se matan"; mientras "Espíritu de la victoria" festejaba actos de resistencia heroicos en los países ocupados por el Eje. A la vez, en cada episodio de "Contraespionaje" se demostraba que los esfuerzos de los espías fascistas para infiltrarse en el hemisferio americano no podían con la inteligencia y moral superiores del contraespionaje estadounidense. Materiales nuevos, producidos exclusivamente para los servicios de onda corta en español y portugués, dramatizaron las guerras de independencia de América Latina y sus héroes como un telón histórico para desarrollar una narrativa que insistió en la necesidad de rechazar nuevamente una agresión europea.

La producción dramática contó con la vasta experiencia de CBS y NBC y en la mayoría de los casos se basó en guiones que habían sido exitosos a nivel nacional. Sin 
embargo, la adaptación de tales géneros para oyentes extranjeros involucraba desafíos difíciles para superar. CBS y NBC contaban con un equipo limitado de personal con capacidades de habla nativa en español y portugués, y muchos de los locutores recién contratados para responder a las necesidades de sus servicios internacionales en plena expansión, llegaron con poca experiencia en la radio. "Justo antes que nada, es hacer mención de la estupenda técnica que predomina... Los efectos de sonido son maravillosos... se percibe en algunas ocasiones hasta el ruido que produce una mosca al emprender su vuelo... La música es inmejorable... ¿ ¿La actuación de los artistas?... Seamos francos [...]" se quejaba un crítico anónimo mexicano. Y luego de detallar algunos de los más atroces errores de pronunciación escuchados en los recientes episodios de producciones dramáticas como "La marcha del tiempo" o "Estamos en Guerra", la crítica concluía: "No... francamente los jóvenes niños o ancianos que toman parte en los programas de onda corta de la N.B.C y de la C.B.S. no tienen idea de lo que es hablar el idioma castellano" ${ }_{29}$.

Más allá de la competencia idiomática de los actores existían otras dificultades a la hora de producir programas para unas regiones vastas con marcadas diferencias en cuanto a acentos y demás idiosincrasias culturales. Los oyentes que habitualmente sintonizaban la onda corta extranjera estaban bien dispuestos a escuchar a voces y acentos foráneos pero los que sintonizaban a sus emisoras favoritas locales para su dosis cotidiana de información y entretenimiento, lo hacían con otras expectativas. En particular, estaban menos dispuestos a soportar programas de entretenimiento y sobre todo de radioteatros presentados con voces extranjeras y hasta con acentos mezclados. Muchos los locutores contratados tenían acentos mexicano y puertorriqueño, lo que provocaba descontento en Sudamérica. Pocos de los críticos contemporáneos fueron tan lejos como el anteriormente mencionado Hernane Tavares de Sa, quien se burló de NBC y CBS por emplear demasiados "latinoamericanos sintéticos de Puerto Rico los cuales se encontraban afanosamente comprometidos [...] con quitar cada vértebra del lenguaje español y transformarla en una ameba babosa” ${ }^{\circ}$. Otros fueron más respetuosos. Benjamín Gache de Radio

29 Radiolandia [México] 24 de noviembre de 1942, p. 10.

$3^{\circ}$ TAVARES DE SÁ, Hernane, The Brazilians. People of Tomorrow, New York, John Day, 1947, pp. 229-30. 
Splendid, en una carta a la NBC, encontró que para los oídos argentinos las voces empleadas en tales programas sonaban bastante "exóticas" ${ }^{1}$.

En Cuba, más que quejas puntuales NBC y CBS se enfrentaron con una rebelión abierta. Las cadenas cubanas, y CMQ en particular, se negaron a continuar con la retransmisión de las series dramáticas que se les ofrecía porque se quejaban de la calidad tonal y dramatúrgica de los programas. Explicaban que el timbre de las grabaciones era demasiado "grave" y el ritmo del tempo tan "lento" que los programas en cuestión "se arrastraban y aburrían" ${ }_{32}^{2}$ En una carta dirigida al gobierno de Estados Unidos, los dueños de CMQ sugerían que toda la campaña propagandística parecía tener poco efecto porque los radioyentes latinoamericanos se sentían "aleccionados" por un poderío prepotente33. Las emisoras cubanas insistían en que la retransmisión de tales programas ponía en peligro sus ratings.

Si bien es cierto que en Cuba como en otras partes existía una buena cantidad de emisoras menores escasas de dinero y con pocos contenidos para diseminar, que se mostraban dispuestas a retrasmitir por una pequeña cuota. Pero estas estaciones no constituían la clientela que la OCAIA, CBS y NBC estaban buscando. Para la OCAIA, y por falta de indicadores más reveladores, el tamaño del público alcanzado por sus programas siguió siendo uno de los criterios principales para medir su impacto. Para las CBS y la NBC, la supervivencia de sus cadenas transcontinentales dependía de su capacidad de construir audiencias masivas que una vez que el apoyo estatal desapareciera con la emergencia de la guerra, iban a atraer un número suficiente de patrocinadores comerciales para equilibrar las cuentas.

\section{Auge y caída de las cadenas panamericanas}

Si los programas producidos por CBS y NBC para la retransmisión en América Latina no despertaron el entusiasmo que la OCAIA había esperado ¿̇por qué entonces tantas emisoras se apuraron a asociarse con las cadenas transcontinentales? En efecto,

\footnotetext{
${ }^{31}$ NARA, RG 229, caja 257, carpeta: Reactions unsolicited, "Benjamin Gache to John F. Royal”, 12 de mayo de 1942, p. 2.

${ }^{2}$ NARA, RG 229, caja 331, carpeta: Reports, "Memorandum R. L. Heydon to Don Francisco", o6 de febrero de 1943, pp. 1-2. La traducción de esta y de las siguientes citas de materiales en inglés pertenece a la autora de este artículo.

33 NARA, RG 229, caja 330, carpeta: Broadcasting Stations, (Misc) July 1, 1943, "Miguel Gabriel and Angel Cambó (CMQ) to Colonel William S. Donovan”, 2 de marzo de 1942, p. 1.
} 
tanto las grandes como las pequeñas emisoras tenían buenas razones para vincularse con las cadenas estadounidenses. Además de conferir cierto brillo a sus negocios, la afiliación con CBS y NBC las ayudaba a conseguir acceso privilegiado a bienes escasos. Para mantenerse al aire, las emisoras dependían del suministro continuo de piezas de repuestos como válvulas muy difíciles de conseguir fuera de Estados Unidos en tiempos de guerra. Como cualquier otra empresa extranjera que solicitara materiales escasos, tenían que solicitar permisos de exportación estadounidenses. Las emisoras afiliadas tenían la ventaja de contar con CBS, NBC y la OCAIA como interlocutores en el proceso burocrático. Dicho soporte podía ser crucial, en particular para los empresarios menores con poca influencia política pero muy dispuestos a cooperar: "Ustedes saben que somos la única emisora en Cuba que, sin falta, retransmite cualquier programa que se nos solicita difundir, incluso si tenemos que cancelar nuestros propios programas importantes”, escribió Manuel Salas, pionero de la radiodifusión y propietario de Radio Salas en una carta solicitando el apoyo de NBC34. Sin embargo, en las condiciones de escasez reinante, incluso empresarios poderosos como Emilio Azcárraga no dejaron de movilizar sus conexiones a la hora de pedir permisos de exportación con las autoridades de Estados Unidos35.

Si tales condiciones proporcionaron un poder notable a Estados Unidos, este poder a la vez tenía sus límites, sobre todo en aquellos contextos donde la OCAIA tenía que lidiar con empresarios fuertes y gobiernos reacios a concederle vía libre al reino de las ondas. Hacia principios de 1943, el optimismo previo de la OCAIA se había esfumado frente a las dificultades crecientes en el manejo de las cadenas Panamericanas. Lejos de encontrar un entusiasmo unánime, los programas producidos por CBS y NBC se enfrentaron con intereses encontrados y las emisoras más importantes de la región estaban aceptando menos de lo esperado, y por buenas razones como reconocía la misma OCAIA. Más allá de la calidad de los programas, había otras razones para rechazarlos.

34 Wisconsin State Historical Society, Madison, WI, Estados Unidos, The NBC Collection (NBC Collection), Royal Papers, caja 110, carpeta 11, "Manuel Salas to Niles Trammell", June 5, 1942.

35 Véase, por ejemplo, los esfuerzos de NBC para conseguir tratamiento prioritario para Azcárraga en: NBC Collection, Royal Papers, caja 110, carpeta 12, "John F. Royal to Don Francisco", 30 de junio de 1942. Sobre los procedimientos de la OCAIA para asegurar "prioridades" ver: "Don Francisco to John F. Royal”, 22 de junio de 1942, carpeta 11. 
En aquellos entornos radiofónicos donde ya existían sindicatos y gremios para defender los intereses de locutores, actores, músicos y demás personal, la importación de altos volúmenes de programas no podía sino causar tensiones. En Argentina, tales tensiones surgieron inmediatamente después de la afiliación de Radio Belgrano con la Cadena de las Américas de CBS. Programas importados desde el exterior, se protestaba, iban a disminuir las oportunidades de trabajo para el talento argentino ${ }^{36}$. En México, mientras tanto, Emilio Azcárraga eligió adelantarse a posibles conflictos con los sindicatos. Por cada programa importado, el magnate de la industria radiofónica les pagó a los gremios de músicos, actores y escritores una cuota para reembolsar a sus miembros la pérdida de ingresos, y cobró a sus socios en Estados Unidos un 50\% adicional sobre sus tarifas regulares por tiempo al aire37. Aun así, la OCAIA reconoció que la situación en México requería de precaución. En otras partes tales tensiones no se habían hecho palpables, cuando los oficiales de la OCAIA se reunieron, en enero de 1943, para revisar el funcionamiento de las cadenas Panamericanas. Tal cual, ellos temían que cualquier esfuerzo para forzar el volumen de las transmisiones hacia arriba, tarde o temprano desembocara en problemas similares 38 .

La OCAIA también reconoció que en varios países, las condiciones políticas reinantes operaban en contra de las cadenas Panamericanas. En Argentina, donde el gobierno conservador de Ramón S. Castillo de 1940-43 se aferró tenazmente a la neutralidad en la guerra, la censura aplicó reglas cada vez más restrictivas para la radiodifusión y tales restricciones se incrementaron aún más después del golpe militar de junio de 1943. Se censuró cualquier intento de cuestionar la política neutralista, y como la programación de la OCAIA, vía CBS y NBC apuntaba a la causa de los Aliados, no sorprende que para las emisoras socias en Argentina retransmitir tales programas implicaba un riesgo creciente. A pesar de su clara orientación pro-Aliada, Radio

\footnotetext{
${ }^{36}$ Véase, por ejemplo, Ray Josephs, "CBS’s Good Neighborliness Seen As Another Shrewd Yankee Trick"; artículo periodístico en: NARA II, RG 229, caja 207, carpeta: propaganda, "John F. Royal to Nelson A. Rockefeller", 30 de enero de 1941; NARA, RG 84, Buenos Aires Embassy Confidential Files 1941, caja 13, carpeta 876, "Confidential Report. Joe D. Walstrom, Further Suggestions as to Radio Programs to Latin America", 14 de marzo de 1941.

37 Emilio Azcárraga to Fred B. Bate, November 18, 1942, NBC Collection, Royal Papers, Box 110, File 12; Memorandum Raleigh Haydon to Don Francisco, November 28, 1942, 2, NARA, RG 229, Entry 1, Box 243, File: Short wave misc (2).

$3^{8}$ NARA, RG 229, caja 258, carpeta: Programs-Rebroadcasts CBS \& NBC "Minutes of Conference held in Washington, January $5^{\text {th }}$ and $6^{\text {th }}, 1943$, to Review the Operations of the Radio Division of CIAA", p. 16.
} 
Belgrano y Splendid, como socios de CBS y NBC, respectivamente se rehusaron cada vez más a retransmitir programas de la OCAIA 39.

En Brasil, las cadenas panamericanas se vieron sofocadas poco después de su concepción. Si bien el gobierno de Getulio Vargas (1930-45) llegó a autorizar a Estados Unidos la construcción y el uso de bases militares en el noreste del país como plataformas para lanzar sus fuerzas aéreas contra el Eje, no estaba dispuesto a ceder control sobre las ondas radiofónicas brasileñas. Vargas permitió la retransmisión de programas producidos en Estados Unidos pero estableció los términos del intercambio radiofónico de una manera que terminó socavando a las cadenas Panamericanas. En pocas palabras: en lugar de permitir a las emisoras brasileñas contratar los programas a retransmitir como socios de CBS y NBC, insistió en una centralización que le dio control sobre los contenidos mismos y su distribución en el país. A su vez, la OCAIA para poder acceder a emisoras brasileñas aceptó incorporar en su equipo a censores vinculados al Departamento de Prensa y Propaganda (DIP) brasilero, posición desde la cual vigilaban la producción de contenidos en lengua portuguesa a ser entregados para su retransmisión a través de Radio Nacional en Rio de Janeiro, la emisora estatal y una de las más importantes radiodifusoras de Brasil. De esta manera, los programas financiados por la OCAIA y producidos en los estudios de CBS y NBC, sobre todo noticieros y programas afines, llegaron a ser retransmitidos en Brasil pero bajo la vigilancia del DIP, un arreglo que pasó por alto los contratos que CBS y NBC habían tratado de establecer por su propia cuenta con emisoras brasileras comerciales ${ }^{40}$.

En otras partes de América Latina, CBS y NBC encontraron un panorama más complaciente. Por ejemplo, en Colombia y Uruguay enfrentaron pocas restricciones estatales en cuanto a retransmisiones de programas por sus asociados locales. En ambos países los gobiernos de turno se alinearon tempranamente con Estados Unidos. Más aun, en ambos países, el paisaje radiofónico se caracterizaba por la existencia de muchas pequeñas emisoras que operando en un mercado publicitario muy estrecho, estaban dispuestas a retransmitir lo que se les ofrecía a poco costo y sin quejarse. Como

39 Véase CRAMER, Gisela, "The Word War at the River Plate" oc. cit.

40 Las particularidades de este y otros acuerdos se discute con más detalle en: CRAMER, Gisela, "Networking the Americas: the Pan-American Radio Networks and the Promise of the American System (1940-48)”, manuscrito inédito (2016). 
vamos a ver más adelante, una emisora uruguaya en particular iba a tener un rol muy importante en las políticas radiofónicas norteamericanas.

Estos socios más complacientes hicieron poco para cambiar la suerte de las cadenas transcontinentales. Para resumir, la Cadena de las Américas (CBS) y la Cadena Panamericana (NBC) no lograron establecer redes comerciales lo suficientemente fuertes para sobrevivir sin el respaldo del Estado, es decir, la OCAIA. Y la OCAIA, aún antes de terminar la guerra, perdió su anterior optimismo acerca de la capacidad de CBS y NBC para anidarse en los paisajes sonoros latinoamericanos. De hecho, hacia comienzos de 1943 sus funcionarios en discusiones internas planteaban el mal funcionamiento de las cadenas Panamericanas y empezaron a expresar su esperanza de que estas "mueran por sí mismas" ${ }^{4}$. Cierto es que, cuando la Cadena de las Américas -la última sobreviviente - dejó de funcionar en julio de 1948, muy pocos observadores de la época se dieron cuenta.

\section{Descentralizando la producción}

El desencanto con las cadenas Panamericanas fue tal que la OCAIA en su afán de conectarse con audiencias masivas, recurrió cada vez más a otra estrategia alternativa: la subcontratación descentralizada, es decir, la producción de programas a nivel local bajo la dirección de sus oficinas sucursales y de agencias de publicidad como J. Walter Thompson o McCann Erickson. Trabajando con elencos de guionistas, locutores y músicos probados a nivel local, la OCAIA siguió el camino de Coca-Cola, Colgate Palmolive, Sterling Products y otras corporaciones que alentadas por nuevas exenciones de impuestas en Estados Unidos, posicionaron crecientes volúmenes de publicidad con emisoras latinoamericanas. Como ya se mencionó antes, las empresas estadounidenses se mostraban reticentes a comprometerse con la onda corta para promover sus productos y servicios en el exterior. En su lugar, invirtieron a nivel local y siguiendo prácticas probadas en Estados Unidos, patrocinaron la producción de programas populares para promover sus marcas comerciales.

De manera similar, la OCAIA buscó fortalecer su presencia en la radiodifusión latinoamericana por medio de un acercamiento a las preferencias culturales regionales. Sin embargo, en comparación con patrocinadores corporativos como los

${ }_{41}$ “Minutes of Conference held in Washington, January 5th and 6th, 1943”, ob. cit, p. 8. 
recién mencionados, la OCAIA promovió la producción de una gama más amplia de contenidos, desde el entretenimiento ligero de "Créase o no" (de Ripley), programa que más allá de los acontecimientos extraños y curiosos que solía tratar, fue usado durante la guerra para ofrecer una imagen favorable de Estados Unidos y su capacidad de ganar la guerra (y la paz), hasta cursos de inglés que animaban a los radioescuchas a enviar sus tareas para que fueran corregidas y calificadas por profesores contratados por la OCAIA. Algunas producciones locales simplemente se montaron sobre guiones estadounidenses o adaptaron formatos bien establecidos tales como la franquicia de Ripley; otras eran contribuciones originales de escritores reconocidos en el medio de la radio de la época como Alejo Carpentier ${ }^{42}$. Entre las producciones locales se destacó la contratación de reconocidos periodistas, intelectuales y diplomáticos, incluyendo a Enrique Santos (Colombia) y Félix F. Palavicini (México), quienes interpretaban las noticias mundiales y el interés nacional en cooperar de manera cercana con Estados Unidos. $\mathrm{Y}$ aunque no todos los programas comisionados por la OCAIA a nivel local resultaron ser éxitos rotundos, por lo general sus ratings parecen haber superado el rango alcanzado por programas similares importados de Estados Unidos43.

La OCAIA dejó una huella muy visible y duradera en una categoría de programación clave, los noticieros. En su afán de "hacer dominantes las noticias [producidas por agencias] estadunidenses" ${ }^{44}$, cooperaba de cerca con la Associated Press (AP) y la United Press (UP), respaldándolas en la búsqueda de nuevos clientes en América Latina. Para tal fin, durante el año 1941 reunió a un número de empresas importantes con intereses comerciales en la región, entre otras, la Standard Oil, para que se hicieran patrocinadoras de noticieros basados en los servicios de AP y UP. Dado el precio de la suscripción, pocas emisoras latinoamericanas habían contratado tales servicios y ahora, en pocos meses, se suscribieron unas 120, con el patrocinio corporativo desde Estados Unidos45. De esta manera, "El reporter Esso" patrocinado por la Standard Oil se estableció firmemente en muchos países incluyendo a Argentina

$4^{2}$ Fragmentos de un libreto escrito para una serie dramática financiada por la OCAIA se encuentran en: LÓPEZ, Oscar Luis, Alejo Carpentier y la radio, La Habana, Letras cubanas, 2003, pp. 160-67.

43 Los mejores sondeos para calibrar el tamaño de las audiencias alcanzados por los programas de la OCAIA se hicieron en México; un resumen de los resultados se encuentra en: ORTIZ GARZA José Luis, La guerra de las ondas, México DF, Planeta, 1992, pp. 252-263.

44 NARA, RG 229, Entry 1, caja 457, carpeta: Francisco, Don, "United States Shortwave in Transition". 45 Véase: NARA, RG 229, caja 256, carpeta: News broadcasts to June 30, 1942, "M. H. Aylesworth to Nelson A. Rockefeller", 9 de diciembre de 1941 y documentos siguientes. 
y Brasil46. Este boletín de noticias empleó un estilo de presentación muy a tono con el medio radiofónico. En vez de oraciones complejas y matizadas, "El reporter Esso" usaba frases cortas, sencillas y asertivas proyectando a la vez una ilusión de imparcialidad y objetividad.

En general, las emisoras latinoamericanas se mostraban receptivas cuando se las invitaba para cooperar en los proyectos locales de la OCAIA. Sin embargo, el balance regional varía con respecto a los volúmenes y contenidos de los programas transmitidos. Sobre todo, fueron los gobiernos y autoridades de radiodifusión locales los que determinaron los límites del campo de acción en el cual se movía la OCAIA. Argentina y Uruguay pueden servir para ejemplificar resultados muy distintos y como veremos ambas experiencias estuvieron íntimamente ligadas.

\section{Argentina}

En Argentina, la OCAIA produjo varias de sus seriales más exitosas que hasta servían como plantillas para ser adaptadas en otras partes de la región. La primera de estas seriales, “Amigos inolvidables", fue emitida en mayo de 1942, es decir, en un momento en que las relaciones entre Argentina y Estados Unidos empeoraban. Estados Unidos, en respuesta a la política de neutralidad argentina empezó a buscar activamente la desestabilización de la economía y del gobierno argentino. 47 Los programas de radio servirían como estrategias complementarias destinadas a inculcar en la opinión pública una visión positiva de Estados Unidos, su pueblo y sus políticas.

Producido por J. Walter Thompson Argentina, la sucursal de una de las agencias de publicidad líderes en Estados Unidos, y emitida por la vasta red de Radio El Mundo, "Amigos inolvidables" estaba inicialmente dedicado a "los grandes Americanos [Estadounidenses] que contribuyeron a un más alto estándar de vida a través de descubrimientos científicos, educativos y prácticos.” 48 Así, el primer episodio

\footnotetext{
${ }^{46}$ Sobre el éxito del noticiero véase, por ejemplo: KLÖCKNER, Luciano "O noticiário radiofónico como política de guerra e a edição brasileira de O Repórter Esso," GOLIN, Cida y BATISTA DE ABREU, Joao (eds.), Batalha sonora o rádio e a Segunda Guerra Mundial, Porto Alegre: EDIPUCRS, 2006, pp. 4972.

47 La literatura sobre el deterioro de las relaciones estadunidenses con Argentina es vasta; véase, por ejemplo, ESCUDÉ, Carlos, Gran Bretaña, Estados Unidos y la declinación argentina 1942-1949, Buenos Aires: Ed. Belgrano, 1988.

${ }^{8}$ NARA, RG 229, caja 1255, Reports [carpeta negra], "Report on Argentine Radio", octubre de 1944, p. 2.
} 
se dedicó a la historia de un grupo de profesores de secundaria estadounidenses quienes a mediados del siglo XIX habían sido invitados para ayudar en la organización y democratización del sistema educativo argentino. Para mediados de 1943, los libretistas aparentemente se habían quedado sin ejemplos históricos para ilustrar su objetivo inicial, y desde allí en adelante se dedicaron a la celebración de Bolívar, San Martín y unos héroes menores destacando su rol en la construcción de una civilización de libertad, propia de las Américas. Aunque cargada con contenidos propagandísticos muy evidentes, la serie parece haber proporcionado una suerte de entretenimiento y aprendizaje que atrajeron la atención de los radioescuchas. También se distribuyeron miles de materiales complementarios gratuitos. Durante los veinticinco meses en que el programa estuvo al aire se recibieron más de 126.000 cartas de radioescuchas de todas partes, solicitando folletos así como 40.000 solicitudes para calendarios con ilustraciones alusivas al programa de radio. La demanda se mantuvo varios meses después de que la serie fuera suspendida por intervenciones cada vez más restrictivas de la censura argentina49.

Mientras "Amigos inolvidables" apuntaba a radioescuchas ávidos de aprender, "Las Andanzas de los Varela" se diseñó para atraer a un público "más popular" ${ }^{\circ}$. Esta radionovela fue escrita por Raúl Gurruchaga, un reconocido guionista de radio y director de cine, y emitida desde diciembre de 1942 en el horario central de Radio Belgrano. Su trama se construyó alrededor de las aventuras del hijo de la familia Varela, Alfredo, quien se encontraba estudiando en Estados Unidos. Sus observaciones sobre la vida en el país del norte, las costumbres y la cultura detalladas en cartas dirigidas a sus seres queridos en Buenos Aires para ser apreciadas y comentadas, servirían como vehículos para la divulgación de una visión positiva de aquel país., Un segundo ciclo se lanzó en abril de 1943, ahora bajo el título "Del brazo con los Varela" y nuevamente, la serie se vio acompañado por materiales gratuitos como tarjetas postales y libretas ilustrando los viajes y encuentros de Alfredo en Estados Unidos. Según los registros de la OCAIA, se recibieron un promedio de 2.174 solicitudes por cada episodio. Los censores argentinos no tardaron en interesarse por las andanzas de los Varela, interfiriendo cada vez más con el guion. Apuntando al

49 Para un análisis más detallado de la serie véase: PRUTSCH Ursula, Creating Good Neighbors? Die Kultur- und Wirtschaftspolitik der USA in Lateinamerika, 1940-1946, Stuttgart, Steiner, 2008, pp. 409-416 y CRAMER, Gisela, "The Word War at the River Plate", ob. cit.

50 "Report on Argentine Radio", octubre de 1944, p. 3, ob. cit. 
corazón de la trama dramatúrgica, finalmente ordenaron que "Alfredo [...] tuviera que regresar a Argentina, y el escenario de acción debe tener lugar en la Argentina" ${ }^{51}$. En julio de 1944, por lo tanto, la serie se suspendió. Cuando dos meses más tarde reapareció, "Del brazo con los Varela" continuó su vida radial bajo patrocinio comercial y despojada de contenidos objetables por las autoridades argentinas ${ }^{2}$.

Otras seriales comisionados por la OCAIA sufrieron un destino similar53. "El reporter Esso" siguió su curso en Radio Belgrano pero ninguno de los programas de mayor afán por adoctrinar a los argentinos logró pasar el control estatal.

\section{Uruguay}

Mientras las relaciones de Estados Unidos con Argentina se empeoraban progresivamente, la OCAIA se fijaba cada vez más en el Uruguay como plataforma para sus actividades propagandísticas en la región del Río de la Plata. Sus sondeos de recepción revelaron que un buen número de frecuencias uruguayas se escuchaban en varias regiones de Argentina, razón por la cual la OCAIA procuró alimentar un volumen creciente de programas, tanto retransmisiones como producciones locales. En Uruguay, los funcionarios de la oficina Rockefeller encontraron poca renuencia cuando se trataba de programar contenidos que "[no malgastaron] ninguna oportunidad para contar la verdad a los argentinos"54, incluyendo "El Cumpleaños de Adolfo Schickelgruber”, programa que se burlaba del Führer y su Reich y que, por esta razón nunca habría pasado la censura argentina55.

Sin embargo, los mismos sondeos de recepción comisionados por la OCAIA sugirieron también que pocos argentinos sintonizaron emisoras uruguayas. Para la OCAIA no hubo gran enigma: la calidad de la oferta radiofónica uruguaya distaba

\footnotetext{
${ }^{51}$ NARA, RG 229, caja 310, carpeta: Radio Reports, "Memorandum 3244, G. F. Granger to Nelson A. Rockefeller", 09.10.1944]

$5^{2}$ Véase: PRUTSCH, Ursula, Creating Good Neighbors? ob. cit., pp. 416-418; CRAMER, Gisela, The World War at the River Plate", ob. cit.

53 "Report on Argentine Radio", Oct. 1944, ob. cit. Más información sobre la censura argentina se encuentra en: NARA, RG 229, caja 310, carpeta: Reports and Surveys, April 1, 1943; "Memorandum 2110, G.F. Granger to Nelson A. Rockefeller”, 22 de noviembre de 1943, p. 3.

54 NARA, RG 229, caja 354, carpeta: Local Committee programs, "Frank Linder to Nelson A. Rockefeller", 22 de octubre de 1943, p. 1.

55 El libreto del programa se encuentra en: NARA, RG 229, caja 353, carpeta: Local Committee Project Authorization, "U-1120 Frank W. Linder to Nelson A. Rockefeller", 28 de abril de 1943.
} 
mucho de la a que estaban acostumbrados los argentinos. Emitidas desde muchas estaciones pequeñas con instalaciones pobres, las ondas uruguayas, aunque audibles, sufrieron interferencias fuertes en el país vecino donde reinaban emisoras con una potencia de transmisión mucho mayor. Además, el nivel de salarios uruguayo simplemente no daba para nutrir talentos de la talla de las estrellas contemporáneas contratadas por empresas como Radio Belgrano, El Mundo o Splendid.

Por lo tanto, la OCAIA buscó mejorar la competitividad uruguaya y para tal fin, envió un equipo de ingenieros y programadores de radio para brindar su ayuda. Sin embargo, como no podía dedicarse con el mismo esfuerzo, a todas las emisoras que competían en el estrecho mercado radiofónico uruguayo, la OCAIA decidió concentrarse, sobre todo, en una emisora: Radio Carve, propiedad de la Sociedad Anónima Difusoras Radioeléctricas del Plata (en adelante SADREP).

Anticipando un empeoramiento progresivo de las condiciones políticas en Argentina, la OCAIA empezó, hacia mediados de 1942, con sus preparativos para convertir a Carve en una megaestación, capaz de jugar un rol dominante en la región del Río de la Plata. Por medio de una empresa subsidiaria clandestina, la que le compró acciones a la SADREP, la OCAIA asumió control mayoritario sobre Carve y sus ingenieros se dispusieron a instalarle un estudio moderno. También prepararon el terreno para antenas y demás instalaciones que fueron importadas con permisos prioritarios de Estados Unidos. Mientras tanto, Frank W. Linder, experto de programación contratado por la OCAIA abordó los contenidos de Carve. Trabajando con los proveedores de noticias, directores de programas, locutores, actores y músicos, se puso a introducir, de manera express, estándares de calidad estadounidenses, pero las cosas no resultaron ser tan fáciles.

Los oficiales de Rockefeller muy pronto se desesperaron por la lentitud con la cual se dio la transformación de Carve. "Quiero dejar constancia en este momento, con una declaración sincera y bien considerada, de que requeriría varios excelentes hombres de radio de Estados Unidos, varios años y una generosa cantidad de dinero en efectivo para llevar esta situación al nivel de las tres grandes [emisoras] en Buenos 
Aires, El Mundo, Belgrano y Splendid", exclamó un colega de Linder en octubre de $1943^{56}$.

La pièce de résistance de todo el proyecto, el nuevo transmisor de $50 \mathrm{KW}$, que pondría Carve a la par con la capacidad instalada de los "tres grandes" de Buenos Aires no se materializó. Enviado de manera urgente desde Estados Unidos aterrizó en las costas uruguayas para quedarse atascado en la aduana. Sujeto a una disputa entre la SADREP y los funcionarios de Aduanas sobre los derechos de importación a pagar, permaneció allí hasta junio de 1944 cuando fue enviado de regreso a Estados Unidos. Para aquél entonces, la OCAIA había renunciado al megaproyecto.57

Más allá de las disputas de aduana, el fracaso del proyecto se debió a una serie de factores, sobre todo políticos. Para empezar, la OCAIA misma perdió fe en su capacidad de transformar a Carve por no contar con los recursos necesarios. No menos importante, la embajada estadunidense en Montevideo llegó a insistir en la terminación del proyecto por temor a las posibles repercusiones a nivel políticodiplomático. A pesar de los esfuerzos en el sentido contrario, la mano de la OCAIA en el entorno radiofónico uruguayo era un secreto a voces. En publicaciones de la oposición política se hablaba de la SADREP llevando un "control imperialista sobre las ondas del aire que significaría la ruina para las estaciones de radiodifusión nacional”, informó la embajada en sus comunicaciones con el Departamento de Estado ${ }^{8}$. También crecía el malestar de los empresarios competidores de la SADREP porque temían por su futuro comercial si se concretaba el megaproyecto 59 . Se informaba también que el gobierno militar al otro lado del Rio de la Plata presionaba, cada vez más, a las autoridades uruguayas para que impidieran el flujo de propaganda política apuntando a Argentina. La embajada propusó retirarse de la SADREP y la OCAIA, además de devolver el transmisor mencionado, revendió sus acciones ${ }^{60}$.

\footnotetext{
${ }^{56}$ NARA, RG 229, caja 573, carpeta No. 4, Uruguayan Radio Project, June 1, 1943- Dec 31, 1943, "Confidential letter Russell to Neave", 23.10.1943, p. 2.

57 Véase CRAMER, Gisela, "The Word War at the River Plate”, ob. cit.

${ }^{8}$ NARA, RG 229, caja 573, carpeta Nro. 5, Uruguayan Radio Project, Jan 1944-June 30, 1944, "Paraphrase of secret telegram Ambassador Dawson to Secretary of State", 28 de junio de 1944, refiriéndose al diario El Debate.

59 NARA, RG 229, caja 573, carpeta No. 5, Uruguayan Radio Project, Jan 1944-June 30, 1944, "Telegram No. 325, Ambassador Dawson to Secretary of State", 1 de abril de1944.

6o Véase CRAMER, Gisela. "The Word War at the River Plate", ob. cit.
} 
Por cierto, la OCAIA no dejó de suministrar un volumen alto de programas a una multitud de emisoras uruguayas, dentro y fuera de Montevideo, incluyendo radionovelas ("La familia William Brown”), música ("Ritmos del soldado americano”), docu-testimoniales ("Mi vida en Berlín”), hasta paneles de comentaristas reconocidos del país discutiendo las noticias actuales (“Tribuna de la prensa”). Lo que no logró fue construir una emisora a la par de las "tres grandes" argentinas para la región del Rio de la Plata.

\section{Para concluir: la radio y el poder en las relaciones interamericanas}

Una revisión de los esfuerzos estadunidenses por influir por medio de la radio a la opinión pública latinoamericana durante la Segunda Guerra Mundial, revela tanto el poder de Estados Unidos para hacerse oír como también los límites de ese mismo poder.

Como hemos visto, durante la Segunda Guerra Mundial Estados Unidos logró afianzar servicios de onda corta potentes con alcance en todos los rincones de América Latina. Las cadenas de CBS y NBC, empresas líderes a nivel mundial estuvieron dispuestas a cooperar con Washington para expandir el sistema radiofónico estadounidense y con ello sus propios intereses empresariales. Más allá de las fronteras de su país lograron afiliar a cientos de emisoras y a muchos de los empresarios latinoamericanos más importantes dentro de sus cadenas transcontinentales. Las agencias estatales creadas para atender emergencias de guerra, y en este caso particular, la Oficina del Coordinador de Asuntos Interamericanos (OCAIA) y sus dependencias a nivel local lograron llenar centenares de horas de programación con contenidos diseñados para apoyar la política exterior de Estados Unidos.

No menos importante, sobre todo en el contexto latinoamericano fue el rol de los grandes patrocinadores comerciales estadounidenses. Si hacia fines de la década de los treinta, empresas como Colgate Palmolive ya contaban con cierta presencia en las radios de Américas Latina como patrocinadores de anuncios comerciales o programas enteros, durante la Segunda Guerra Mundial el gobierno estadunidense procuró ahondar la dependencia financiera de los medios de comunicación latinoamericanos, como hemos visto, por nuevas exenciones de impuestos. Por último, 
es necesario recordar que Estados Unidos en la coyuntura particular de la Segunda Guerra Mundial contaba con una ventaja excepcional: podía rechazar los permisos de exportación para productos esenciales en el funcionamiento de las emisoras latinoamericanas, como válvulas, que difícilmente se podía conseguir en otras partes.

En vista de semejantes asimetrías de poder se podría pensar que las políticas radiofónicas estadunidenses tuvieran un impacto más bien uniforme sobre los paisajes sonoros latinoamericanos. Sin embargo, no fue así. Cierto es que la onda corta estadunidense terminó distribuyéndose más o menos por igual por todos los rincones de América Latina, pero en sus esfuerzos de alcanzar audiencias masivas por medio de emisoras latinoamericanas, las cadenas radiofónicas y agencias estadounidenses se enfrentaron con unos entornos bien distintos que podían reforzar o delimitar su capacidad de maniobra. Tales esfuerzos, como hemos visto en las páginas anteriores, dependían en última instancia de la colaboracíon o por lo menos la acceptación pasiva de empresarios y autoridades locales, y esta colaboración o aceptación no ocurrieron de igual manera en todas partes.

Los hallazgos de este ensayo apuntan a la relevancia de enfoques teóricos y agendas de investigación más recientes que si bien reconocen la naturaleza profundamente asimétrica de las relaciones interamericanas insisten en la necesidad de tomar más en serio la "agencia" latinoamericana para entender mejor el impacto de la política exterior estadunidense en la región ${ }^{61}$. Sin embargo, asignando tal agencia no implica necesariamente que, con ella, podamos prescindir del imperialismo como marco de análisis. En última instancia, aún los imperialistas más reacios en la historia de las imperios, sabían tolerar, y hasta aprovecharse, de alguna medida de la agencia subalterna cuando se trataba de imponer sistemas duraderos de subordinación en América Latina y otras partes del mundo.

\footnotetext{
61 Para un sondeo de las tendencias historiográficas al respecto véase, por ejemplo: HARMER, Tanya "Commonality, Specificity, and Difference: Histories and Historiographies of the Americas", SCARFI, Juan, et al. (eds.), Cooperation and Hegemony in US-Latin American Relations: Revisiting the Western Hemisphere Idea, New York, Palgrave Macmillan, 2016.
} 


\section{Bibliografía}

BOELCKE, Willi A., Die Macht des Radios. Weltpolitik und Auslandsrundfunk, 19241976 Frankfurt am Main, Ullstein, 1977.

CHILDS, Harwood L., "Shortwave Listening in the United States". Public Opinion Quarterly 5, June 1941, pp. 210-226.

CRAMER, Gisela, "Der Krieg im Äther: Radiopropaganda am Río de la Plata, 19391945", Holger MEDING y Georg ISMAR (eds.), Argentinien und das Dritte Reich, Berlin, Wissenschaftlicher Verlag, 2008, pp. 151-168.

CRAMER, Gisela, "Networking the Americas: the Pan-American Radio Networks and the Promise of the American System (1940-48)", manuscrito inédito, 2016.

CRAMER, Gisela, "The Word War at the River Plate: The Office of Inter-American Affairs and the Argentine Airwaves, 1940-46." Gisela CRAMER y Ursula PRUTSCH (eds.) iAméricas Unidas! Nelson A. Rockefeller's Office of InterAmerican Affairs, 1940-46, Frankfurt y Madrid, Vervuert, 2012.

ESCUDÉ, Carlos, Gran Bretaña, Estados Unidos y la declinación argentina 19421949, Buenos Aires, Ed. Belgrano, 1988.

FEJES, Fred, Imperialism, Media, and the Good Neighbor: New Deal Foreign Policy and United States Short Wave Broadcasting to Latin America, Norwood, NJ, Ablex, 1986.

FRIEDMAN, Max Paul, Nazis and Good Neighbors: The United States Campaign Against the Germans of Latin America in World War II, Cambridge, Cambridge University Press, 2003.

GREGORY, Bruce N., The Broadcasting Service: An Administrative History. The United States Information Agency Special Monograph Service, Washington, DC, USIA, 1970.

HARMER, Tanya, "Commonality, Specificity, and Difference: Histories and Historiographies of the Americas", Juan SCARFI et al. (eds.), Cooperation and Hegemony in US-Latin American Relations: Revisiting the Western Hemisphere Idea, New York, Palgrave Macmillan, 2016.

KLÖCKNER, Luciano, "O noticiário radiofónico como política de guerra e a edição brasileira de O Repórter Esso," Cida GOLIN y Joao BATISTA DE ABREU (eds.), Batalha sonora o rádio e a Segunda Guerra Mundial, Porto Alegre, EDIPUCRS, 2006. 
LOPÉZ, Oscar Luis, Alejo Carpentier y la radio, La Habana, Letras cubanas, 2003. LÜBKEN, Uwe, Bedrohliche Nähe. Die USA und die nationalsozialistische Herausforderung in Lateinamerika, 1937-1945, Stuttgart, Steiner, 2004.

MORENO, Julio, Yankee Don't Go Home: Mexican Nationalism, American Business Culture, and the Shaping of Modern Mexico, 1920-1950, Chapel Hill, NC, University of North Carolina Press, 2003.

OGILVIE, John W. G., “The Potentialities of Inter-American Radio,” Public Opinion Quarterly 9, 1 (spring 1945), pp. 19-28.

ORTIZ GARZA, José Luis, La guerra de las ondas, México DF, Planeta, 1992.

PRUTSCH, Ursula, Creating Good Neighbors? Die Kultur- und Wirtschaftspolitik der USA in Lateinamerika, 1940-1946, Stuttgart, Steiner, 2008.

RABE, Robert, "Selling the Shortwaves: Commercial Broadcasting to Latin America and the Limits of the 'American System'," in American Journalism 24:4, pp. 127-48, 2007.

TAVARES DE SA, Hernane, The Brazilians. People of Tomorrow, New York, John Day, 1947.

\section{Fuentes}

BBC Year Books

National Archives and Record Administration, College Park, MD, Estados Unidos (NARA), Record Groups 59, 84, 229

Radiolandia [México]

Rockefeller Archive Center (RAC), Tarrytown, Estados Unidos, RFA, Record Group 4, NAR Papers, Washington Files

The New York Times, Estados Unidos

The Washington Post, Estados Unidos

Wisconsin State Historical Society, Madison, WI, Estados Unidos, The NBC Collection (NBC Collection), Royal Papers 